\title{
Noninvasive Neural Prostheses Using Mobile and Wireless EEG
}

\author{
A system using micro-electro-mechanical sensors has been used to \\ detect motion sickness and help participants maintain alertness \\ while using an automobile driving simulator.
}

\author{
By Chin-Teng Lin, Fellow IEeE, Li-Wei Ko, Jin-Chern Chiou, Member IEeE, \\ Jeng-Ren Duann, Member IEeE, Ruey-Song Huang, Sheng-Fu Liang, \\ Tzai-Wen Chiu, and Tzyy-Ping Jung, Senior Member IEEE
}

\begin{abstract}
Neural prosthetic technologies have helped many patients by restoring vision, hearing, or movement and relieving chronic pain or neurological disorders. While most neural prosthetic systems to date have used invasive or implantable devices for patients with inoperative or malfunctioning external body parts or internal organs, a much larger population of "healthy" people who suffer episodic or progressive cognitive impairments in daily life can benefit from noninvasive neural prostheses. For example, reduced alertness, lack of attention, or poor decision-making during monotonous, routine tasks can have catastrophic consequences. This study proposes a noninvasive mobile prosthetic platform for continuously monitoring high-temporal resolution brain dynamics without requiring application of conductive gels on the scalp. The proposed system features dry microelectromechanical system electroencephalography sensors, low-power signal acquisition, amplification and digitization,
\end{abstract}

Manuscript received April 20, 2007; revised February 12, 2008. This work was supported by the National Science Council, Taiwan, R.O.C., under Contracts NSC 96-2627-E-009-001 and NSC 96-2218-E-009-001; the "Aiming for the Top University Plan," National Chiao-Tung University; the Ministry of Education, Taiwan, under Contract 96W803; and the Defense Advanced Research Projects Agency under Grant NBCH1060010.

C.-T. Lin, L.-W. Ko, and J.-C. Chiou are with the Brain Research Center and Department of Electrical and Control Engineering, National Chiao-Tung University, Hsinchu, Taiwan, R.o.C. (e-mail: ctlin@mail.nctu.edu.tw; Iwko@mail.nctu.edu.tw; chiou@mail.nctu.edu.tw).

J.-R. Duann and T.-P. Jung are with the Brain Research Center, National Chiao-Tung University, Hsinchu, Taiwan, R.O.C. They are also with the Institute for Neural

Computation, University of California San Diego, La Jolla, CA 92093 USA (e-mail: duann@sccn.ucsd.edu; jung@sccn.ucsd.edu).

R.-S. Huang is with the Institute for Neural Computation, University of California San Diego, La Jolla, CA 92093 USA (e-mail: rshuang@sccn.ucsd.edu).

S.-F. Liang is with the Brain Research Center, National Chiao-Tung University, Hsinchu, Taiwan, R.O.C. He is also with the Department of Computer Science and Information Engineering, National Cheng-Kung University, Tainan, Taiwan, R.O.C.

(e-mail: sfliang@mail.ncku.edu.tw).

T.-W. Chiu is with the Brain Research Center, National Chiao-Tung University, Hsinchu, Taiwan, R.o.C. (e-mail: twchiu@mail.nctu.edu.tw; tzaiwen.chiu@gmail.com).

Digital Object Identifier: 10.1109/JPROC.2008.922561 wireless telemetry, online artifact cancellation, and signal processing. Its implications for neural prostheses are examined in two sample studies: 1) cognitive-state monitoring of participants performing realistic driving tasks in the virtualreality-based dynamic driving simulator and 2) the neural correlates of motion sickness in driving. The experimental results of these studies provide new insights into the understanding of complex brain functions of participants actively performing ordinary tasks in natural body positions and situations within real operational environments.

KEYWORDS | Cognitive-state monitoring; electroencephalography (EEG); motion sickness; neural prosthesis; noninvasive mobile prosthetic platform

\section{INTRODUCTION}

Prosthesis refers to a surrogate or replacement of a body part such as a tooth, an eye, a facial bone, an ear, a hip, a knee or other joint, a leg, or an arm. Prostheses may be designed for functional or cosmetic purposes or both. Advances in biomedical science and technology have enabled integration of some prostheses with body tissues, including the central nervous system. These neuroprostheses can now respond to commands from the brain. During the current decade, hundreds of thousands of patients have already been helped by neuroprosthetic technologies that restore vision [1], hearing [2] or movement [3] and relieve chronic pain or neurological disorders [4]. These neuroprostheses usually involve invasive or implantable devices for patients with inoperative or malfunctioning external body members or internal body organs [5]. However, a much larger population of "healthy" people who suffer momentary, episodic, or progressive cognitive impairments in daily life have, ironically, been overlooked. For 
example, as the last 60 years of research in human vigilance and attention has shown, humans are not well suited for maintaining alertness and attention under monotonous conditions, particularly during the normal sleep phase of their circadian cycle [6]. Catastrophic errors can result from momentary lapses in alertness and attention during periods of relative inactivity. Many people, not just patients, can benefit from a prosthetic system that continuously monitors fluctuations in cognitive states in the workplace and/or the home. However, to be practical for routine clinical or occupational use, the prosthetic system must be noninvasive, nonintrusive, lightweight, battery-powered, and easy to don and doff. Further, it must enable a full range of head, eye, and body movements. The only possible brain-imaging modality fulfilling these criteria is electroencephalography (EEG). EEG is a powerful noninvasive tool widely used for both medical diagnosis and neurobiological research because it can provide high temporal resolution in milliseconds that directly reflects the dynamics of the generating cell assemblies. Electroencephalography is also the only brain-imaging modality that can be performed without fixing the head/body. Substantial research [7]-[11] has shown that many features of EEG dynamics index the current state of subject alertness, arousal, and attention. However, data collection in most EEG studies requires skin preparation and gel application to ensure good electrical conductivity between sensor and skin. These procedures are time consuming, uncomfortable, and even painful for participants since skin preparation usually involves abrasion of the outer skin layer. Repeated skin preparation and gel application for EEG may also cause allergic reactions or infections. Further, the signal quality may degrade over time as the skin regenerates and the conductive gel dries. Advance electrode designs are needed to overcome these requirements and complications of adhesive contacts between EEG electrodes and the skin surface before routine EEG monitoring can be feasible in real-world environments.

Another major challenge for EEG monitoring in operational environments is extracting meaningful and informative event-related brain dynamics from the recorded signals that are often subject to severe artifacts from head/body motion or other ocular or muscle movement. The recorded information generally has value only in proportion to the sophistication and reliability of the analytical method employed. Sophisticated signalprocessing techniques are often computationally expensive and implemented either online or offline using high-end personal computers (PCs). The substantial processing requirements hinder the wearability, portability, and practical use of the systems in operational environments. However, given the recent development of embedded system and signal-processing techniques, it is now practical to implement these sophisticated algorithms in embedded systems for online EEG monitoring and/or brain-computer interface (BCI). These real-time embed- ded systems, in conjunction with wireless transmission, have proven useful in applications such as diagnosis and homecare systems [12] because they provide maximum portability and wearability.

In short, the unavailability of EEG monitoring systems that do not require application of conductive gels to the scalp and are capable of high-definition recording, online signal processing, and artifact cancellation has long thwarted applications of EEG monitoring in other than well-controlled laboratory conditions. To make the noninvasive prosthetic system practical for routine use, it is essential to provide a quickly and easily donned and doffed EEG acquisition system for implementing online signalprocessing techniques to continuously and accurately extract meaningful and informative information. The system should also be a convenient size, rugged, and lightweight and have low power consumption to meet the requirements of wearability, portability, and durability.

This study details the design, development, and testing of a noninvasive mobile prosthetic platform for continuously monitoring high-temporal resolution brain dynamics without requiring conductive gels applied to the scalp. The system employs dry microelectromechanical system (MEMS) EEG sensors, low-power signal acquisition, amplification and digitization, wireless telemetry, online artifact cancellation, and real-time signal processing. Its implications in neuroprostheses are also demonstrated through two sample studies: 1) cognitive-state monitoring of participants performing realistic driving tasks in a virtual reality-based dynamic driving environment and evaluation of the efficacy of the system in delivering stimulating feedback to help maintain optimal task performance and 2) identification of neural correlates of motion sickness in driving.

\section{NONINVASIVE PROSTHETIC SYSTEM}

The study reported in this paper designed and developed a noninvasive mobile prosthetic platform (Fig. 1) consisting of self-stabilized MEMS sensors, signal amplification and digitization, wireless transmission/receiving, and a realtime embedded system to assess human physiological and mental information in operational environments.

\section{A. Self-Stabilized MEMS Sensor}

Electrodes for biopotential measurement transduce biosignals from skin tissue to the amplifier circuit. They are used extensively in biomedical applications to measure biosignals such as EEG and electrocardiogram (ECG) signals [13]. The most important task in designing and fabricating the biopotential electrode is ensuring low electrode/skin interface impedance so that the measured biosignals can be bridged into the front-end circuits with minimal attenuation and noise interference [13]. Skin anatomy can be divided into three layers: the epidermal, dermal, and subcutaneous layers [14]. As Fig. 2(a) shows, 


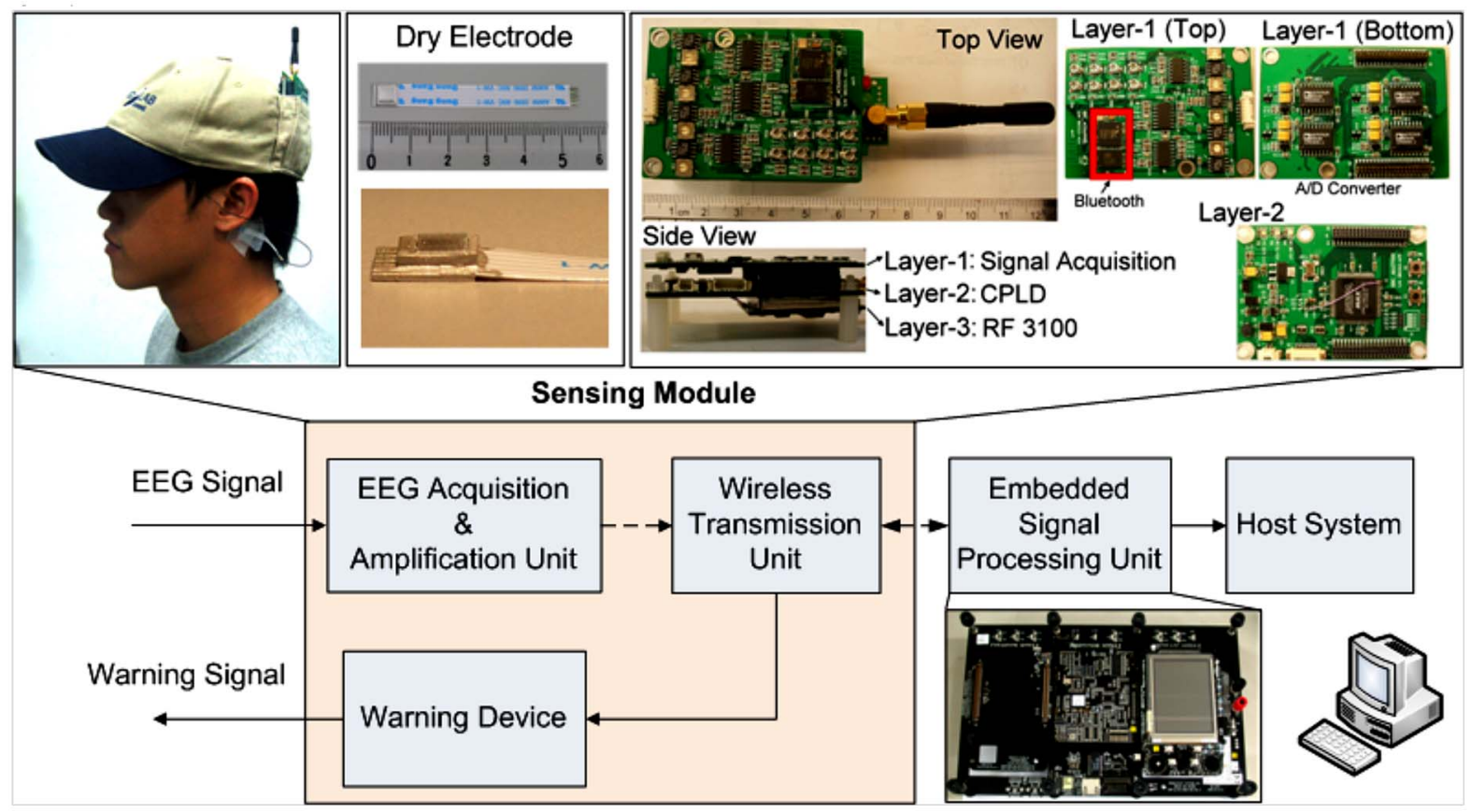

Fig. 1. The system diagram of the proposed noninvasive prosthetic system.

the epidermis contains two layers: stratum corneum (SC) and stratum germinativum (SG). The SC consists of dead cells and thus has electrical isolation characteristics. The SG is composed of living cells and is therefore electrically conductive. The dermis contains nerve endings, blood vessels, and oil glands. To overcome the electrical isolation property of the $\mathrm{SC}$, conventional wet electrodes always require skin preparation (abrasion on SC) and the use of an electrolyte. Improper preparation can cause skin irritation, pain, or even infection. Using electrolytic gel on the skin tissue not only is uncomfortable and inconvenient but also causes irritation and swelling during long-term measurement. Further, the conductivity of electrolytic gel decreases gradually due to drying. The decreasing conductance can in turn degrade signal quality.
For practical use of biomedical signal monitoring in the workplace, collecting the biosignals using easily donned and doffed dry electrode technology is highly desirable. In recent years, the authors have explored the use of MEMS technology to build a silicon-based spiked electrode array or so-called dry electrode array to enable EEG, electrooculograph, ECG, and magnetoencephalogram [13] monitoring without conductive paste or scalp preparation. The dry MEMS sensors feature a microprobe array structure and self-stabilization capability. Microprobe arrays placed on the skin provide signal transmission performance superior to that of standard wet electrodes. Transmission is improved because the electrically conductive probe can penetrate the high electrical resistance of the outer SC skin layer and into the deeper SG skin layer, which has better electrical conductivity [15]. As Fig. 2 shows, the

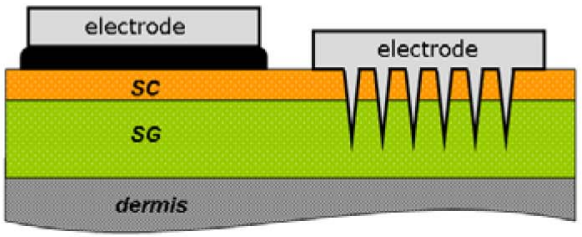

(a)

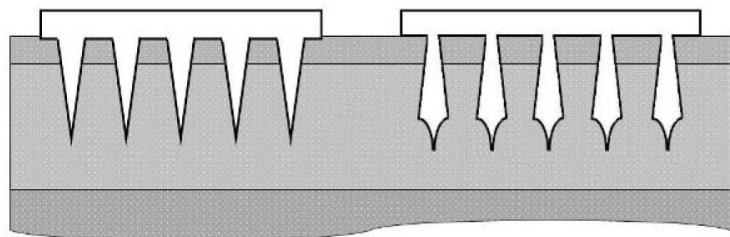

(b)

Fig. 2. Skin anatomy: comparison between (a) conventional wet electrode, conical shape electrode and (b) proposed self-stabilized MEMS EEG sensor. 


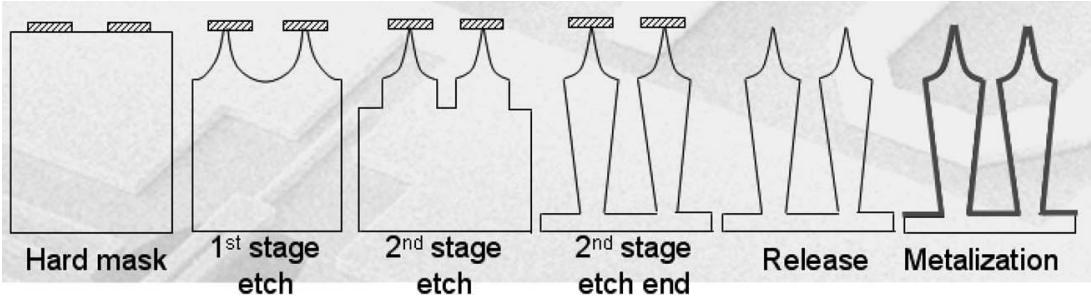

Fig. 3. Fabrication process of the proposed self-stabilized microprobe array.

electrically conductive probes are designed to avoid the dermal layer so as to avoid pain and bleeding. Since the proposed sensor is expected to bypass the high impedance of the SC skin layer, skin preparation and electrolytic gel application are no longer required. In contrast with other designs such as that proposed in [13], this study develops a simple and stable technology for fabricating EEG sensors.

1) Design and Fabrication: Fig. 3 shows the silicon-based process for microfabrication of the self-stabilized diamondshaped microprobe. The most important parameter in the fabrication process is the probe length, which should be sufficient to pierce the SG layer but not the dermis. The thickness of the epidermis varies from 0.05 to $1.5 \mathrm{~mm}$ in different ethnic groups and in different areas of the body, and the thickness of SC and SG layers is approximately 10-15 and 50-100 $\mu \mathrm{m}$, respectively [16]. Thus, to penetrate the SC and reach the SG layers, the length of the probe must be longer than $20 \mu \mathrm{m}$ and the tip must be sufficiently sharp to minimize damage during penetration. Fig. 4 shows a scanning electron microscope image of the fabricated electrodes. The dimensions of the manufactured probe are $\mathrm{a}=17 \mu \mathrm{m}, \mathrm{b}=50 \mu \mathrm{m}$, $\mathrm{c}=200 \mu \mathrm{m}$, and $\mathrm{d}=50 \mu \mathrm{m}$. The tip of the probe is slightly blunt $(<10 \mu \mathrm{m})$ because the tip must support the hard mask in the second anisotropic etching.

2) Testing and Evaluation: To test the electrode/skin interface impedance, two dry electrodes were placed on the forehead $4 \mathrm{~cm}$ apart, and electrode/skin impedance was measured after applying current to the electrode pair [17]. The measured impedance was then compared to that obtained from wet electrode pair with the same setup. Nineteen tests were performed on five different subjects. Five different electrodes were used: two standard wet electrodes coated with $\mathrm{AgCl}$ and $\mathrm{Au}$ of $1 \mathrm{~cm}$ diameter and three fabricated MEMS electrodes with sizes of $4 \times 4$, $3 \times 3$, and $2 \times 2 \mathrm{~mm}^{2}$. Fig. 5(a) shows the impedance for a frequency range $(0.5-150 \mathrm{~Hz})$ obtained by dry electrode pair (red trace) without skin preparation or conducting gel, with $\mathrm{AgCl}$ electrode (green trace), and without applying gel (blue trace). Electrode/skin impedances for five different electrodes ( $\mathrm{Au}, \mathrm{AgCl}, 4 \times 4,3 \times 3$, and $\left.2 \times 2 \mathrm{~mm}^{2}\right)$ were evaluated without using skin preparation or electrolytic gel. As Fig. 5(b) shows, the electrode/skin impedances of all the MEMS electrodes were lower than those of the wet electrodes without preparation in almost all frequency ranges. These experimental results indicated that the MEMS electrodes outperformed the conventional (wet) electrodes in terms of electrode/skin interface impedance and signal intensity [18].

\section{B. Mobile and Wireless Noninvasive \\ Prosthetic System}

The study reported in this paper proposed a noninvasive prosthetic system using mobile and wireless EEG comprising the above MEMS EEG sensors, a signal acquisition and amplification unit, wireless telemetry,
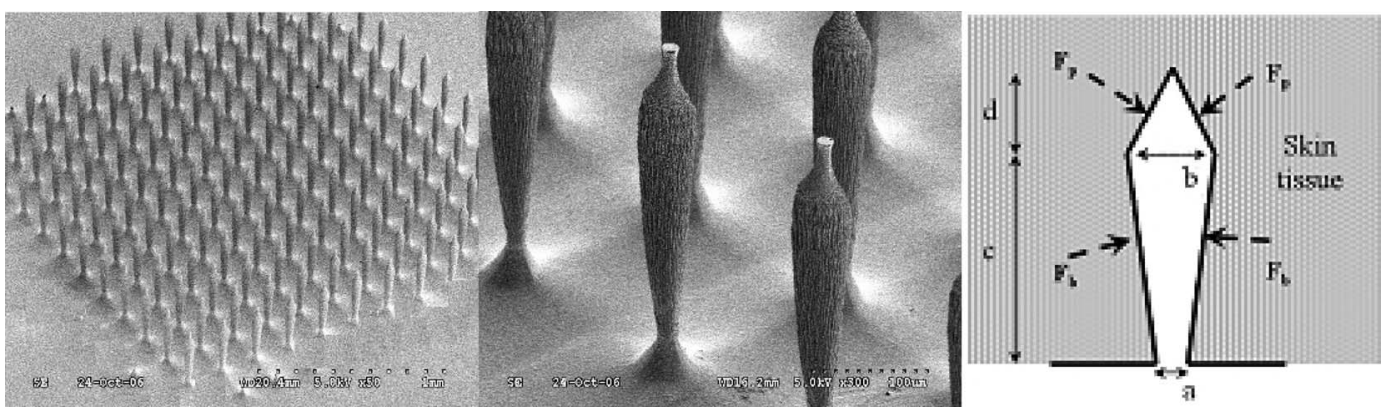

Fig. 4. The fabrication result of the proposed design. The dimension of the microprobe is about $\mathbf{a}=17 \mu \mathrm{m}, \mathrm{b}=50 \mu \mathrm{m}$, $\mathbf{c}=\mathbf{2 0 0} \mu \mathrm{m}$, and $\mathbf{d}=\mathbf{5 0} \mu \mathrm{m}$ 


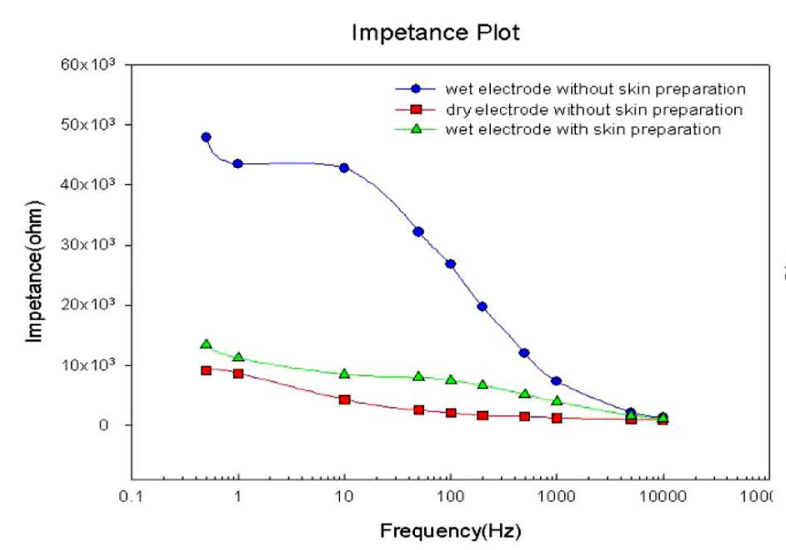

(a)

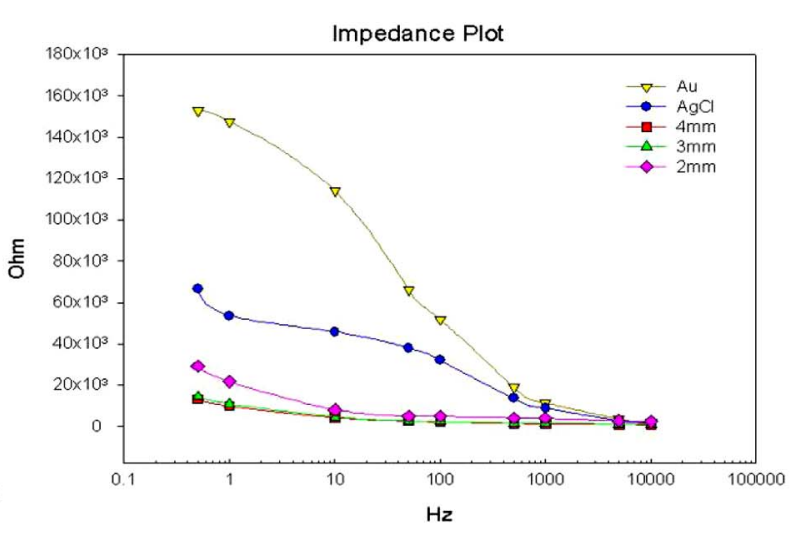

(b)

Fig. 5. Comparisons of impedance spectra for the test biopotential electrodes.

and online signal processing modules. The system analyzes EEG signals in near real-time to monitor human cognition states and delivers arousing feedback to help maintain subject alertness. The left side of Fig. 6 shows the detailed system architecture of the noninvasive prosthetic system, which consists of five major units: (a) signal acquisition and amplification units, (b) wireless data transmission unit, (c) dual-core processing unit, (d) host system for data storage and real-time display, and (e) warning device. The EEG signals acquired by MEMS electrodes are first amplified by the signal acquisition and amplification unit. The wireless data transmission unit consists of an analog-to-digital converter, a complex programmable logic device, and a wireless module. The wireless module can be either a radio-frequency ( $R F)$ module (RF3100/3105) or a Bluetooth module depending on transmission range requirements. The heart of the mobile and wireless noninvasive prosthetic system is a dual-core processing unit based on Texas Instruments' Open Multimedia Architecture Platform 1510 featuring an ARM925 processor and a
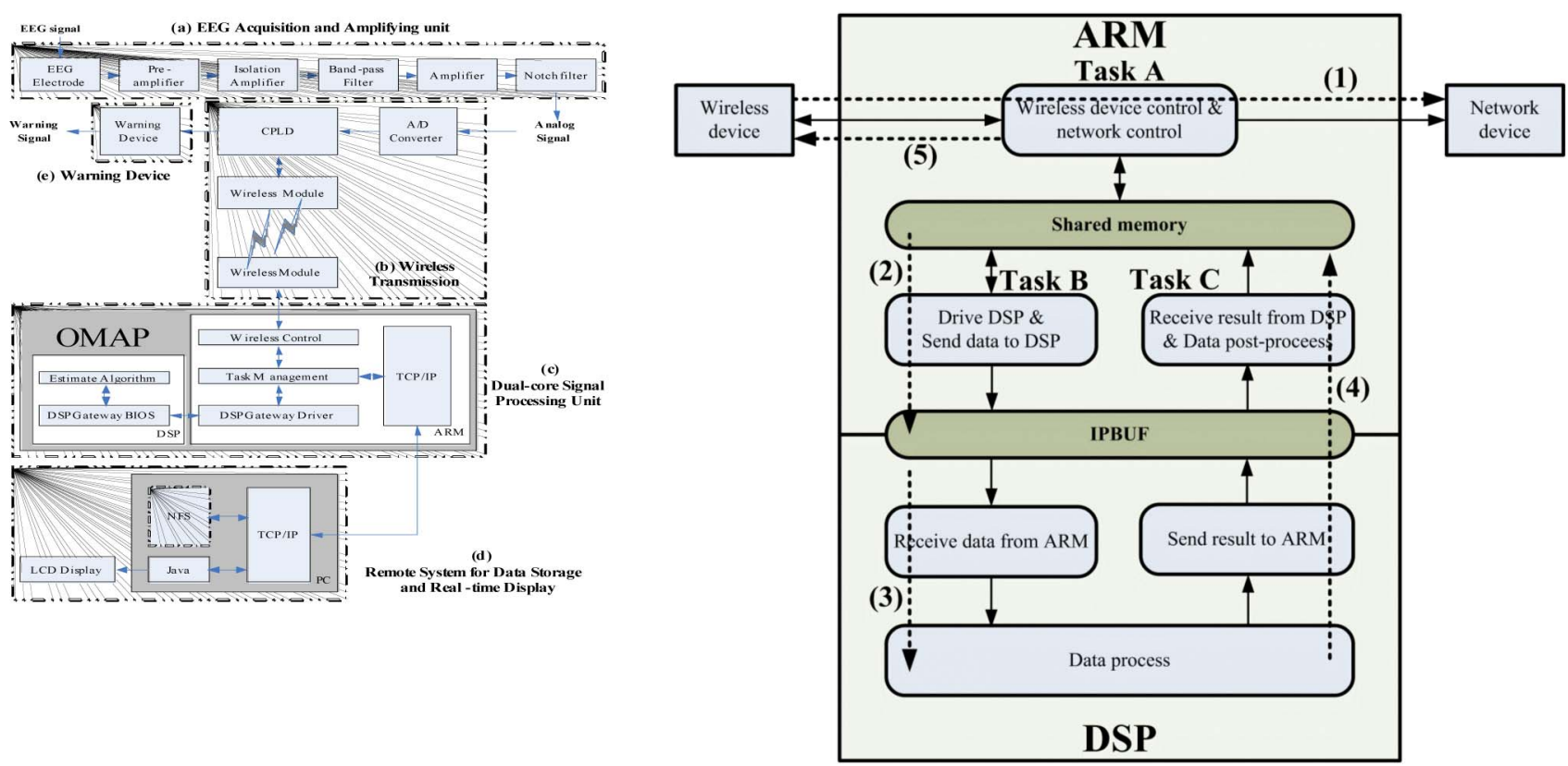

Fig. 6 (Left) Detailed system architecture of the mobile and wireless prosthetic system. (Right) The structure of the embedded system and the data processing flowchart. 
TMS320C55x digital signal-processing (DSP) processor. The DSP core is used to process EEG data, and the ARM925 is used to communicate with other devices such as wireless transmission modules and TCP/IP networks. The DSP gateway software enables the ARM core to use DSP resources by application program interface (API) and functions similarly to a small real-time kernel, which manages resources and data flow in the DSP core. Therefore, it is used as the cooperation structure for communication between the two cores since these two cores perform different functions. This mechanism activates the DSP processor only when the system must process EEG data. The Linux operating system (OS) is used to manage core ARM resources. The three components of the ARM are 1) wireless module control, 2) TCP/IP control and 3) DSP gateway driver. The right side of Fig. 6 shows the process flowchart and task distribution in the embedded system.

The host system is equipped with data storage and realtime EEG signal display functions. The connection between the host and embedded systems uses TCP/IP protocol. The warning device is also incorporated in the system. Visual and/or audio signals can be delivered to users to provide stimulating feedback signals. A multitasking and scheduling procedure is employed in the dual-core signal processing unit to enhance the efficiency of the embedded system and to enable to the system to function in near real-time [19]. The unique features of the proposed system are:

1) novel dry electrodes that noninvasively and nonintrusively acquire EEG signals without requiring skin preparation or conductive gel;

2) a miniaturized and battery-powered signal acquisition and digitization module for maximum portability;

3) a selectable wireless transmission module that maximizes wearability and can be adapted to many other applications;

4) a dual-core embedded system with a multitasking and scheduling procedure for online signal processing and artifact cancellation;

5) a feedback delivery device that provides stimulating feedback to help users optimize task performance.

\section{DEVELOPMENT OF KINESTHETIC VIRTUAL-REALITY DRIVING ENVIRONMENT}

To demonstrate the implications of the noninvasive prosthetic system, declines in the cognitive state of participants were monitored in a sustained-attention task. Driving is one of the most common attentiondemanding tasks in daily life. Driver fatigue or drowsiness is reportedly a major causal factor in many accidents because sleepy drivers have markedly reduced perception, recognition, and vehicle control abilities. In recent years, technology for preventing driving accidents caused by drowsiness has become a major interest in automotive safety engineering. Physiological changes such as eye activity measures, heart rate variability, and particularly EEG activity are known to be associated with drowsiness [20]-[25].

Most previous studies of drowsiness measured the psychophysical responses while participants performed a computer-simulated driving task in a well-controlled laboratory in which driving controls were a joystick or a steering wheel in front of a computer screen. In such studies, behavioral data such as reaction time to perform sudden braking or turning maneuvers are often measured. However, participants are never provided with kinesthetic input or sensation during these experiments. The lack of multisensory (visual, auditory, kinesthetic, and tactile) information presented to participants limits the assessment and interpretation of the complicated relationships between such information and the motor actions of the participants. The ideal experimental condition would measure physiological signals in an actual automobile on the road. However, investigating driving perception in an actual driving environment would be subject to several limitations. First, ethical concerns would prohibit exposing subjects to physical danger of attention lapse while driving an automobile. Secondly, appropriate data acquisition and monitoring devices are needed to study the rapid physiological responses of kinesthetic stimuli. Simulators provide simple and repeatable stimulation to control the parameters of the experiment. Thirdly, objective evaluation of driving performance and putative level of alertness may be difficult to assess on the road. To solve such problems of studying kinesthetic perceptions during driving, a realistic simulator is the best alternative for driving-related research [26]-[29].

To test and demonstrate the feasibility of a noninvasive prosthetic system in a realistic operational environment, the study reported in this paper developed a threedimensional (3-D) virtual-reality (VR) dynamic motion simulator [30], [31] as a test bed for studying the EEG dynamics associated with cognitive-state changes during driving. The simulator provides visual, auditory, and, most importantly, kinesthetic and tactile stimulation either separately or jointly to volunteer subjects, which are not available in conventional EEG laboratories. This dynamic driving simulator enables systematic testing of the limitations of normal human performance in sustainedattention tasks in a safe yet realistic environment. Seven projectors are used to project $360^{\circ}$ highway and driving scenes in 3-D [Fig. 7(a)]. For the study, an actual automobile was mounted on a six-degree-of-freedom (DOF) Stewart platform [Fig. (7b)]. The 6-DOF motion platform is controlled by six hydraulic linear actuators to generate translational and rotational movement as well as vibratory feedback to simulate actual driving conditions. 


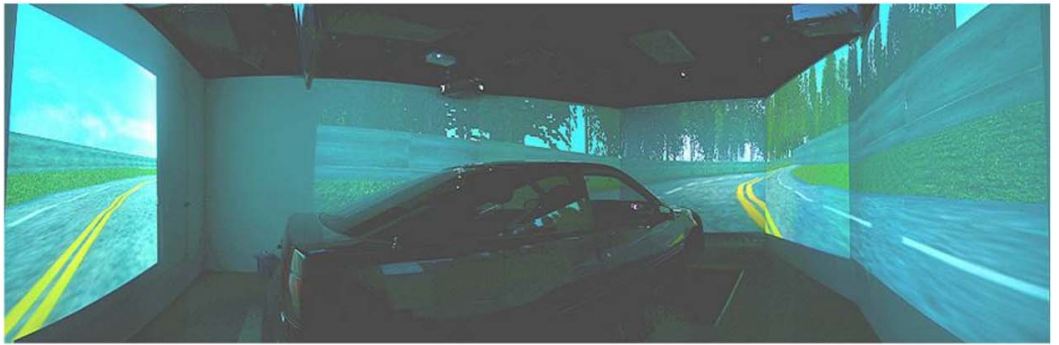

(a)

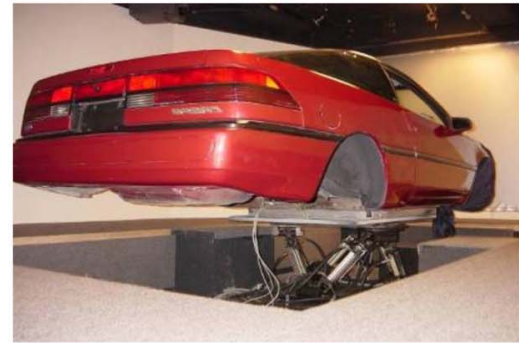

(b)

Fig. 7. (a) Kinesthetic VR-based driving environment; (b) driving cabin simulator mounted on a 6-DOF dynamic Stewart motion platform.

The $360^{\circ}$ projection of driving scenery is updated synchronously with deviations caused by wheel/paddle movement by the subjects or by road conditions such that subjects feel the car moving as if they are driving in realworld conditions. The motion platform also provides physiological and behavioral response recordings to not only to evaluate driving performance and behavior but also examine the brain dynamics in response to the kinesthetic stimulation generated by the motion platform. Therefore, this test environment provides an interactive, safe, and realistic environment at very low cost, and the outcomes of the study should be highly applicable to real-life driving safety research.

\section{CASE STUDY: DROWSINESS DETECTION IN LONG-TERM DRIVING}

The goal of the study was to examine the efficacy of a noninvasive prosthetic system for monitoring declines in cognitive function during a sustained-attention task. By combining EEG power spectrum estimation, principal component analysis (PCA), and linear regression of artificial neural networks, the authors previously reported that continuous, accurate, noninvasive, and near real-time estimation of alertness of an operator is feasible [30], [31]. The study employed dry MEMS sensors and mobile wireless EEG monitoring technologies to extend previous laboratory studies of EEG-based drowsiness detection to a simulated operational environment using a realistic kinesthetic VR driving simulator.

\section{A. Method and Materials}

Ten subjects (aged 20-40 years; mean 29.8 years; standard deviation \pm 5.9 ) participated in the VR-based highway driving experiments, which were all conducted in the early afternoon after lunch. The VR scenes simulated driving at a constant speed $(100 \mathrm{~km} / \mathrm{h})$ on a highway with the car randomly drifting away from the center of the cruising lane to simulate driving on nonideal road surfaces or with poor alignment [32]. Other than a straight and monotonous road, no traffic or other stimuli appeared in the VR scene, which was intended to simulate a driving situation likely to induce drowsiness.

All participants completed informed consent forms before being briefed on the task requirements. All subjects practiced keeping the car in the cruising lane using the steering wheel for 15-30 min until they reached a performance asymptote for the task. After the practice session, conventional wet electrodes and MEMS EEG sensors were prepared and placed on the forehead of each subject (Fig. 8), who then performed the driving task for 1 h. Each participant returned on a different day to complete a second 1-h driving session. Driving performance was measured by the distance of lane deviation, which was small when the subject was alert, and vice versa. The driving parameters (lane position and wheel rotation) were in sync with the EEG acquisition system.

Fig. 8 shows the placements of the MEMS/conventional electrode pairs at the five forehead locations. The first and fifth MEMS EEG sensors were placed at Fp1 and Fp2 according to the international 10-20 electrode placement system [33]. Three additional MEMS EEG sensors were also evenly positioned between these two MEMS sensors

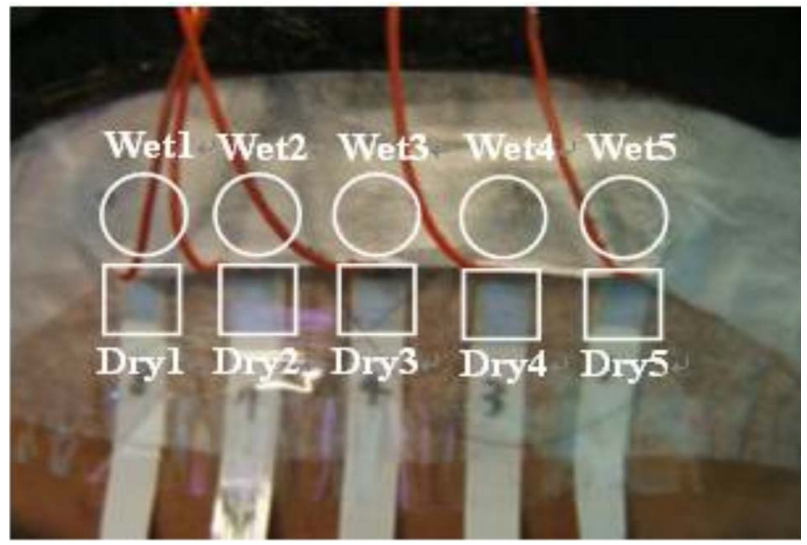

Fig. 8. Forehead positions of (circle) conventional wet electrodes and (square) MEMS EEG sensors. 
and labeled as MEMS2, MEMS3, and MEMS4. Corresponding conventional wet electrodes were placed $1 \mathrm{~cm}$ above the MEMS EEG sensors. The contact impedance between the MEMS/wet electrode and skin tissue was calibrated to less than $5 \mathrm{k} \Omega$. The EEG signals were recorded from these five MEMS and five wet sensors, then referenced against linked mastoids (A1, A2) by the proposed noninvasive prosthetic system and Neuroscan NuAmps Express system (Compumedics Ltd., VIC, Australia), respectively. The frequency range was selected to bandpass from 0.5 to $100 \mathrm{~Hz}$ with a $60-\mathrm{Hz}$ notch filter, recorded with 16-bit quantization level at a sampling rate of $500 \mathrm{~Hz}$ and downsampled to $250 \mathrm{~Hz}$ to simplify signal processing.

\section{B. Results}

Fig. 9 plots the raw EEG signals measured by the noninvasive mobile wireless prosthetic system and traditional EEG system (only the leftmost and rightmost MEMS/wet pairs are shown). The figure shows that the EEG signals recorded by the MEMS sensors are virtually identical to those obtained by the corresponding wet electrodes. Fig. 10 overplots the EEG power spectra of EEG data collected by five MEMS/wet electrode pairs. The plot shows their similarity, especially in low frequency bands (1-30 Hz), indicating that EEG signals obtained by the proposed noninvasive prosthetic system using MEMS sensors approximated those recorded by the conventional EEG system using conventional wet electrodes.

Fig. 11 shows data analysis procedures for estimating the drowsiness level from EEG power spectra. The acquired EEG signals were fed into an EEG-based drowsiness estimation program [31] to estimate the driver drowsiness level during long-term driving. First, artifacts were rejected before further analysis. A low-pass filter with a cutoff frequency of $50 \mathrm{~Hz}$ was applied to EEG data to remove line noise and other high-frequency noises. The time series of recorded behavior data (driving performance) and EEG signals were smoothed using a causal 90-s square moving-averaged filter advancing at 2-s steps to eliminate variance at cycle lengths shorter than 1-2 min since driving performance becomes erratic at cycle lengths of 4 min and longer [8], [9]. After moving-average power spectral analysis, an EEG log power spectrum was obtained for the five MEMS (or wet) electrodes. Logarithmic scaling linearizes the expected multiplicative effects of subcortical systems involved in wake-sleep regulation on EEG amplitudes [34]. Karhunen-Loeve PCA was then applied to the resultant EEG log spectrum between $1-40 \mathrm{~Hz}$ to extract the directions of largest variance for each session. Finally, projections of the EEG log spectral data (PCA features mapping) along the subspace formed by the eigenvectors corresponding to the largest 50 eigenvalues were used as inputs to a multiple linear regression model [35] to estimate the time course of driving error for each subject [36]. The features of each model were trained and extracted only from the training session and tested on the data from a separate testing session.

Figs. 12-15 compare the estimation performance obtained by MEMS EEG sensors and wet electrodes in the sustained-attention driving tasks. In each figure, the blue and red traces represent the acquired and estimated driving errors, respectively, and all of the figures show test data results. Fig. 12(a) and (b) shows the estimated driving error for Subject 1 in Session \#2 using the EEG signals recorded by the conventional wet electrodes and the MEMS EEG sensors, respectively. The estimators were trained with the EEG signals from Session \#1 to estimate the driving errors in Session \#2 of Subject 1 (the blue traces in Fig. 12). Conversely, Fig. 13(a) and (b) shows the estimated driving error of Subject 1 using EEG data from Session \#2 as the training dataset and those from

\section{Raw EEG Recording}

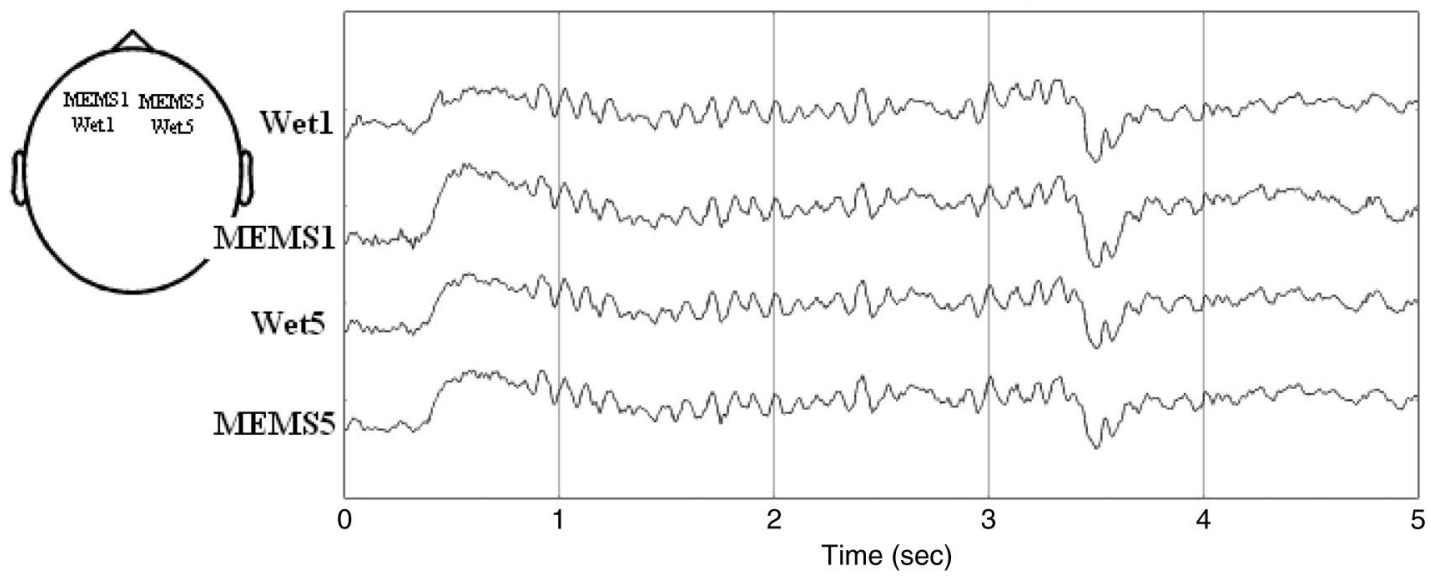

Fig. 9. EEG signal recording contrast MEMS sensors with wet electrodes. 


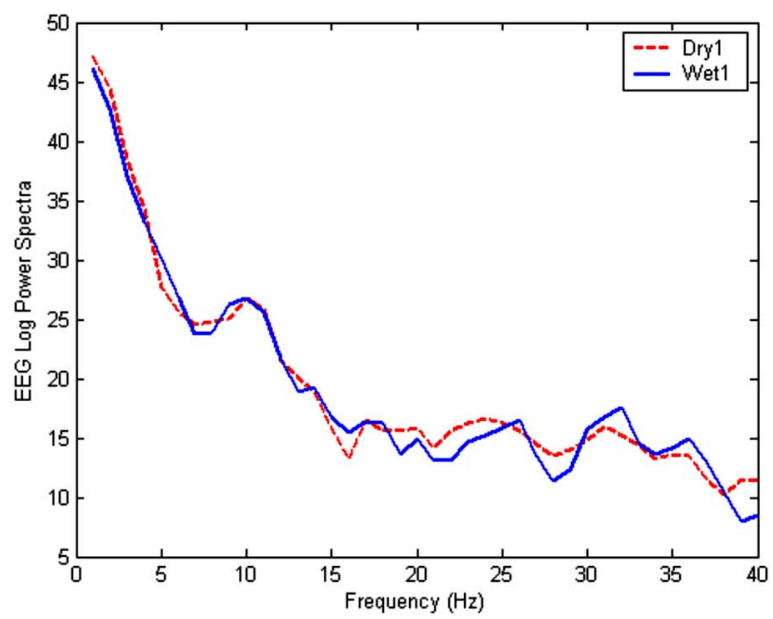

(a)

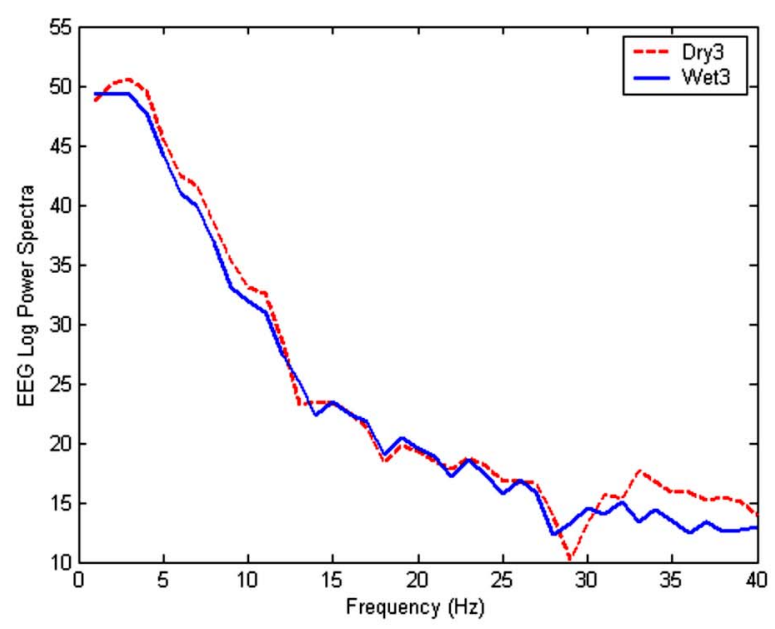

(c)

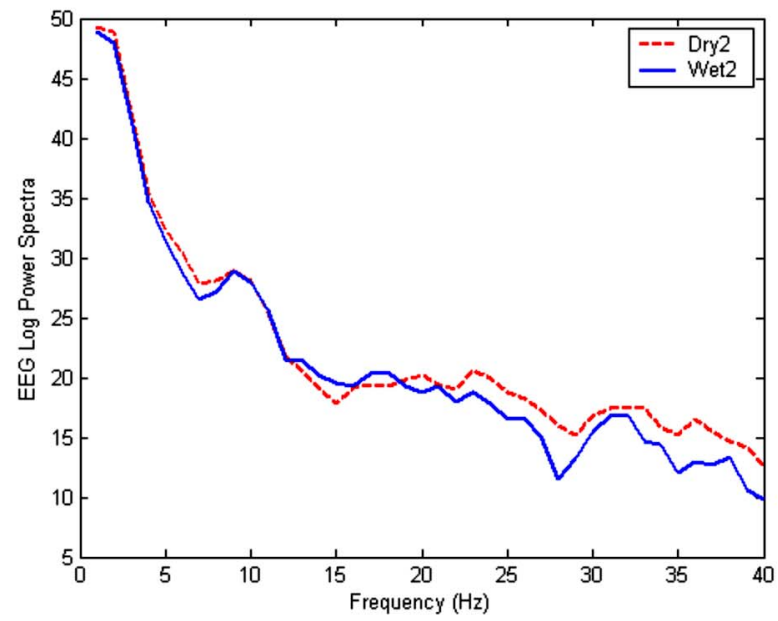

(b)

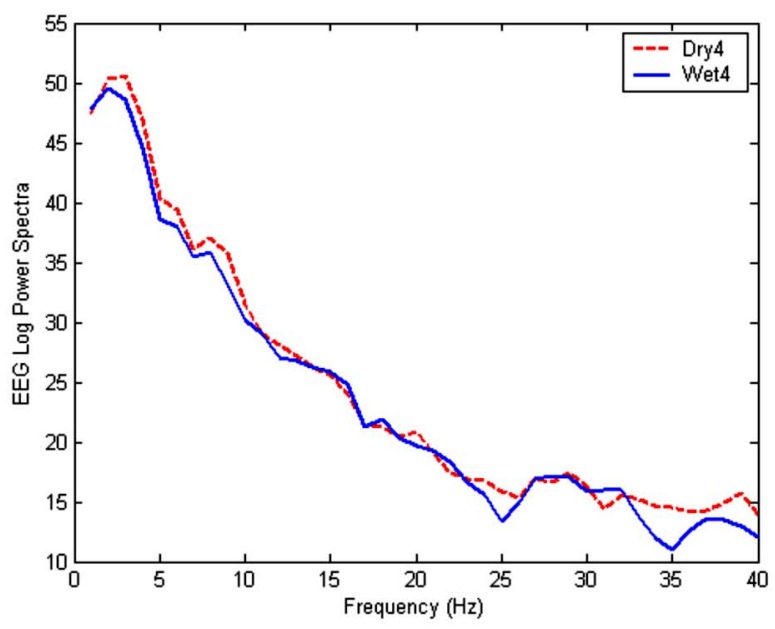

(d)

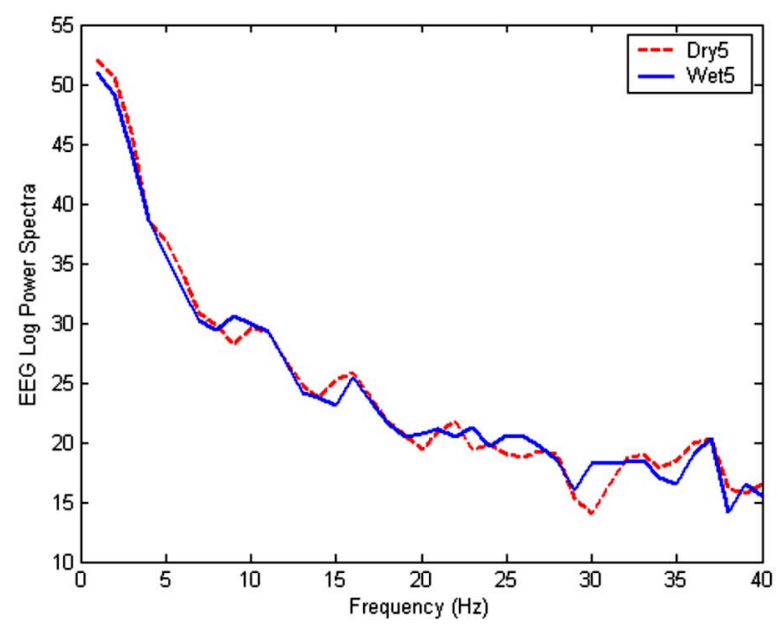

(e)

Fig. 10. Comparison of EEG power spectra between five dry (MEMS)/wet electrode pairs. (a) Dry1 (MEMS1)/Wet1 electrode pair. (b) Dry2 (MEMS2)/Wet2 electrode pair. (c) Dry3 (MEMS3)/Wet3 electrode pair. (d) Dry4 (MEMS4)/Wet4 electrode pair. (e) Dry5 (MEMS5)/Wet5 electrode pair. 


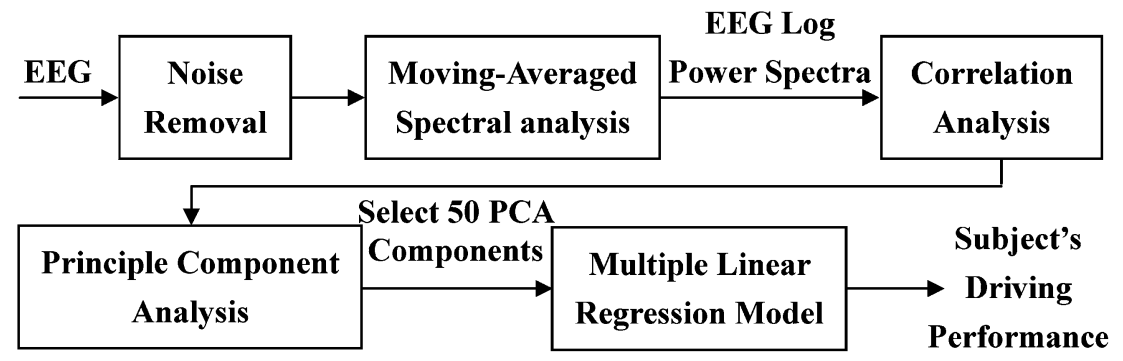

Fig. 11. Flowchart for processing the EEG signals. 1) A low-pass filter was used to remove the line noise and higher frequency (>5O Hz) noise. 2) Moving-averaged spectral analysis was used to calculate the EEG log power spectrum of each channel advancing at 2-5 steps. 3) Two EEG channels with higher correlation coefficients between the subject's driving performance and EEG log power spectrum were further selected. 4) PCA was trained and used to decompose selected features and extract the representative PCA components as the input vectors for the linear regression models. 5) The linear regression models were trained in one training session and used to continuously estimate and predict the individual subject's driving performance in the testing session.

Session \#1 as the testing dataset. Similarly, Figs. 14 and 15 show estimated and actual driving errors made by another subject (Subject 2). Table 1 compares correlation coefficients between the actual and estimated driving error time series using MEMS EEG sensors and conventional wet electrodes for ten different subjects. As Figs. $12-15$ and Table 1 show, the estimated driving errors based on EEG spectra matched well with actual errors, which was consistent with a recent report by the authors in which the same driving tasks were analyzed by whole-head 32-channel EEG [30], [31]. These analytical results demonstrate the feasibility of accurately estimating subject task performance based on EEG signals collected by dry MEMS sensors positioned on frontal areas. Further, the estimation accuracy based on the EEG collected by the MEMS EEG sensor is comparable to that of signals collected by conventional wet electrodes, which indicates that the proposed noninvasive prosthetic system does not require skin preparation or conductive gels to acquire highquality EEG signals in operational environments.

\section{CASE STUDY: MOTION SICKNESS WHILE DRIVING ON WINDING ROADS}

Motion sickness can be induced when humans are exposed to vestibular or visual motion stimuli. Symptoms may differ according to the vehicle or environment, such as car sickness, sea sickness, air sickness, and space sickness. Motion sickness can induce symptoms including eye strain, headache, pallor, sweating, vertigo, ataxia, nausea, and vomiting. Many previous studies indicate that motion sickness can sometimes impair cognitive and response

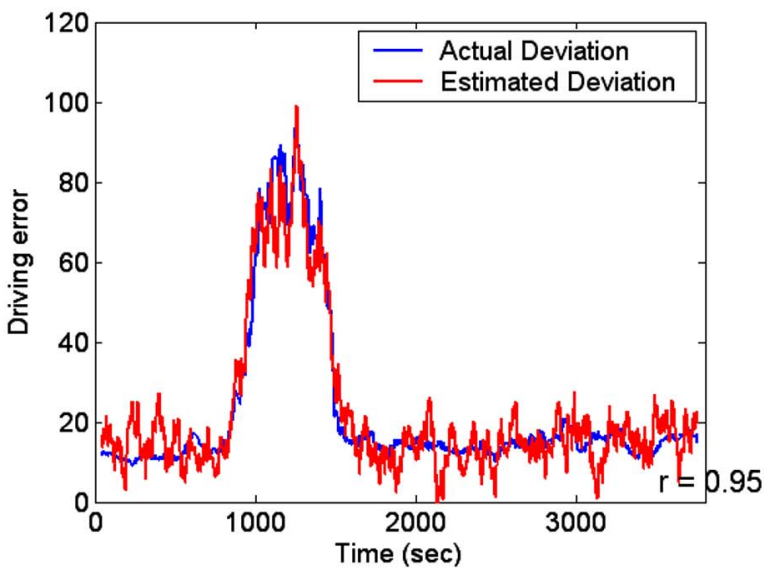

(a)

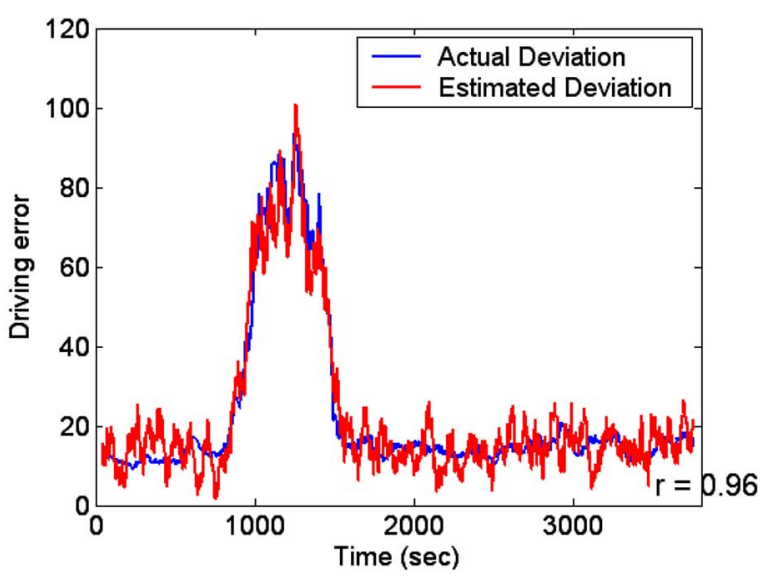

(b)

Fig. 12. Estimated (red traces) and actual (blue traces) driving error of Session \#2 of Subject 1 using the EEG signals recorded by (a) the conventional wet electrodes and (b) MEMS EEG sensors, respectively. The estimators were trained with the EEG signals from Session \#1 to estimate the driving error of Session \#2. 


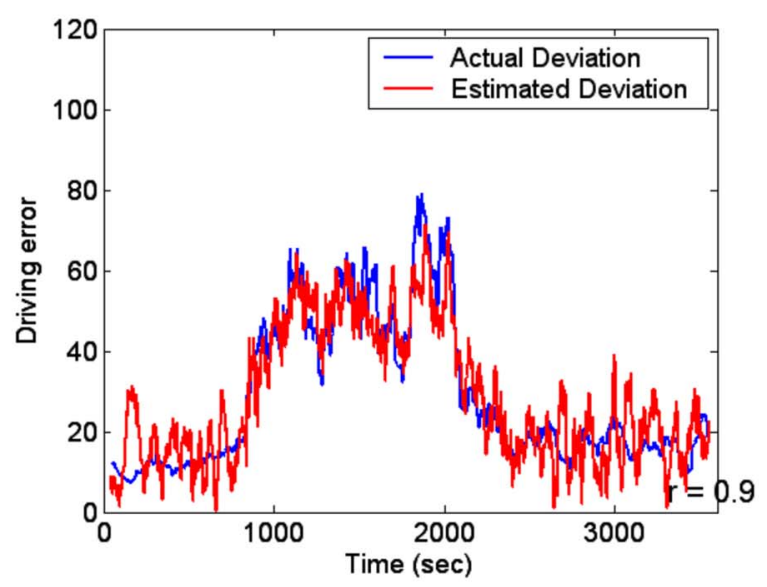

(a)

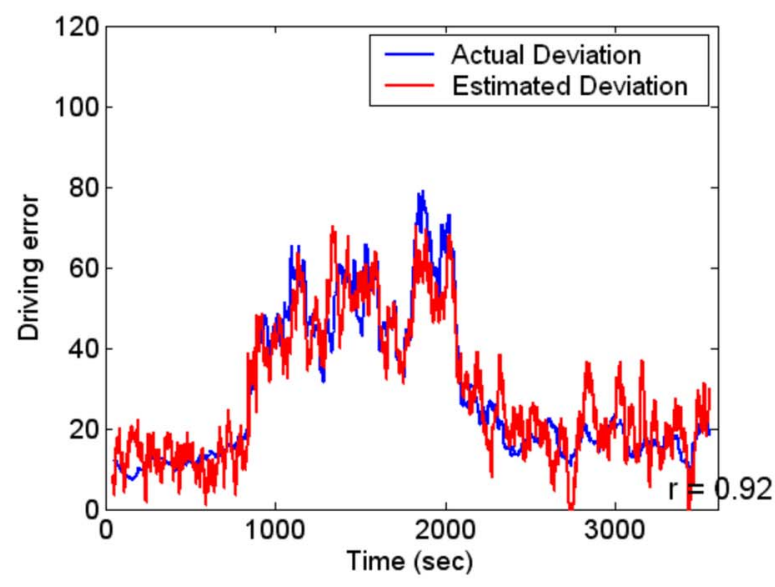

(b)

Fig. 13. Estimated (red traces) and actual (blue traces) driving error of Session \#1 Subject 1 using the EEG signals recorded by (a) wet and (b) MEMS electrodes, respectively. The estimators were trained with the EEG signals of Session \#2 to estimate the driving error of Session \#1.

ability [37], [38]. Thus, numerous studies have attempted to elucidate motion sickness and its symptoms. Two main theories of the cause of motion sickness are the sensory conflict theory [39] and the postural instability theory [40].

Studies have employed various methods of inducing motion sickness, such as rotary chair [41], circular vection drum [42], and off-axis yaw oscillator [43]. According to the sensory conflict theory, subjects get sick in a virtual environment because of the conflict between the visual and vestibular system. Thus, conflict can be induced or reduced by using a motion platform in a VR environment. The syndrome of sickness should be induced or totally eliminated if the motion of the platform is misaligned or perfectly aligned with the VR scene, respectively. The goal of the study reported in this paper was to demonstrate the potential use of a noninvasive prosthetic system for predicting the onset of motion sickness while driving by exploring the EEG correlates of motion sickness systematically induced/reduced by the aforementioned kinesthetic VR driving platform.

\section{A. Method and Materials}

A three-stage driving task was designed. Each subject was given a 10-min practice session before each experiment to familiarize himself with the VR environment and vehicle controls. Each experiment started with a 10-min

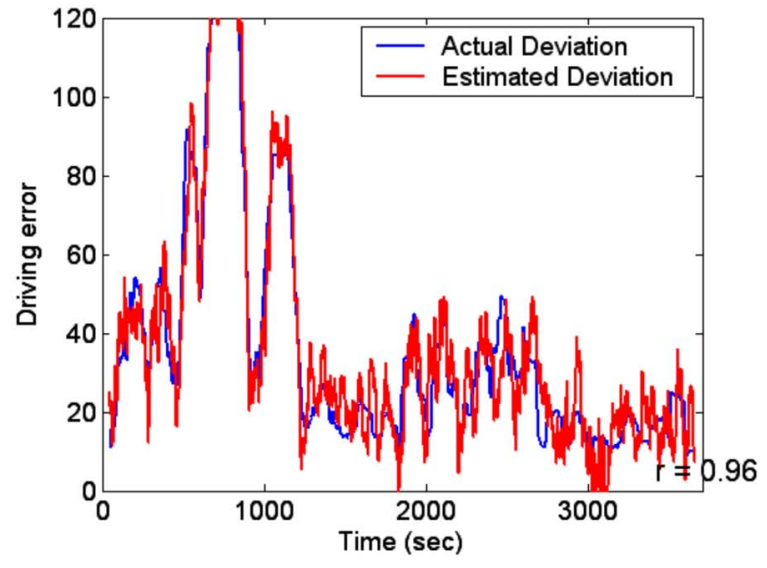

(a)

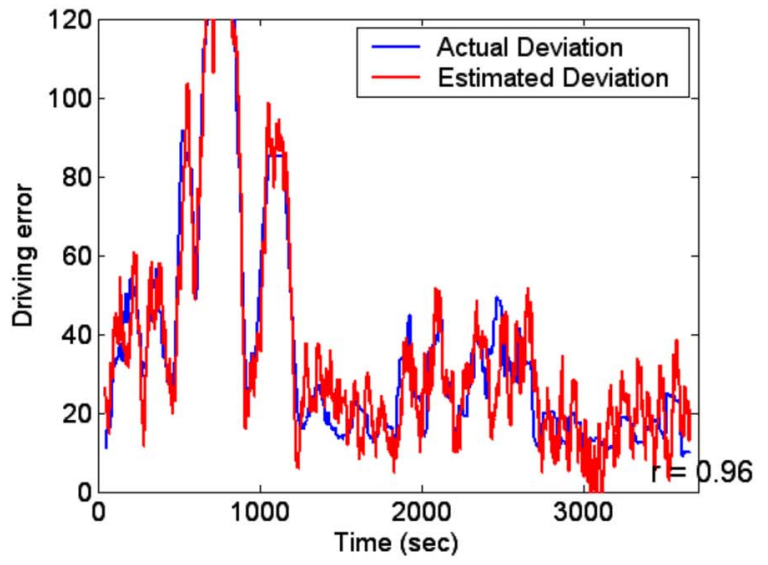

(b) 


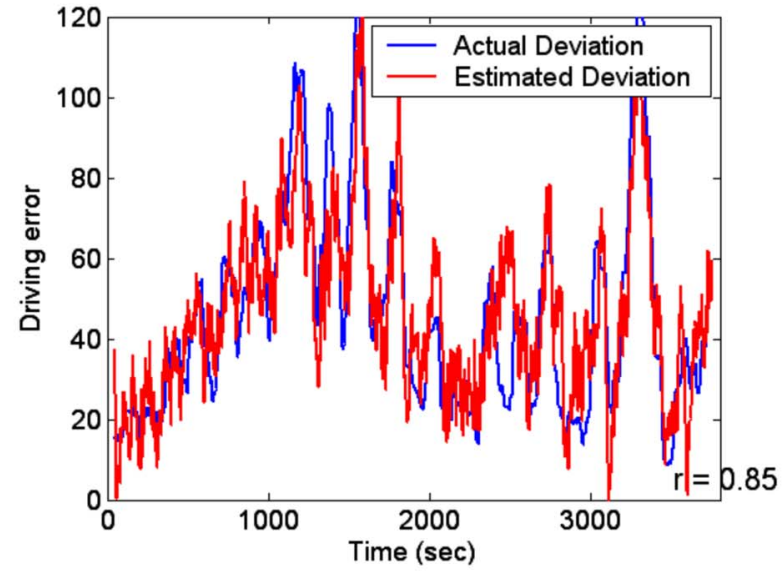

(a)

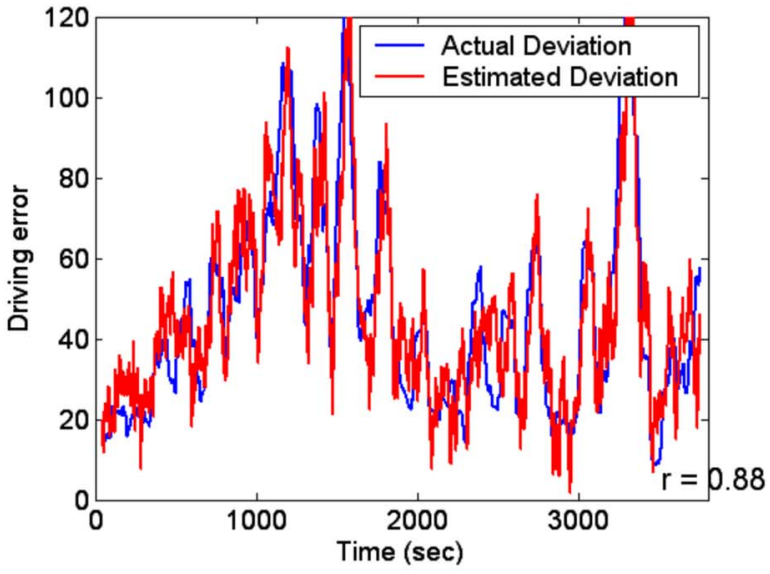

(b)

Fig. 15. Estimated (red traces) and actual (blue traces) driving error of Session \#1 of Subject 2 using the EEG signals recorded by (a) wet and (b) proposed electrodes, respectively. The estimators were trained with the EEG signals of Session \#2 to estimate the driving error of Session \#1.

Table 1 Testing Patterns for Electrode-Skin-Electrode Impedance (ESEI) Measurement

\begin{tabular}{|lcccc|}
\hline \hline & \multicolumn{2}{c}{ Session 1 estimates Session 2 } & \multicolumn{2}{c|}{ Session 2 estimates Session 1 } \\
\cline { 2 - 5 } & Wet electrodes & $\begin{array}{c}\text { MEMS EEG } \\
\text { sensors }\end{array}$ & Wet electrodes & $\begin{array}{c}\text { MEMS EEG } \\
\text { sensors }\end{array}$ \\
\hline Subject 1 & $95 \%$ & $96 \%$ & $90 \%$ & $92 \%$ \\
\hline Subject 2 & $96 \%$ & $96 \%$ & $85 \%$ & $88 \%$ \\
\hline Subject 3 & $82 \%$ & $83 \%$ & $84 \%$ & $86 \%$ \\
\hline Subject 4 & $92 \%$ & $94 \%$ & $81 \%$ & $83 \%$ \\
\hline Subject 5 & $85 \%$ & $86 \%$ & $85 \%$ & $88 \%$ \\
\hline Subject 6 & $93 \%$ & $92 \%$ & $91 \%$ & $90 \%$ \\
\hline Subject 7 & $87 \%$ & $88 \%$ & $86 \%$ & $85 \%$ \\
\hline Subject 8 & $81 \%$ & $83 \%$ & $90 \%$ & $90 \%$ \\
\hline Subject 9 & $92 \%$ & $90 \%$ & $86 \%$ & $87 \%$ \\
\hline Subject 10 & $88 \%$ & $88 \%$ & $86 \%$ & $85 \%$ \\
\hline Mean & $89.1 \%$ & $89.6 \%$ & $86.4 \%$ & $87.4 \%$ \\
\hline \hline
\end{tabular}

period of straight road driving followed by $40 \mathrm{~min}$ of winding-road driving to induce motion sickness, followed by $15 \mathrm{~min}$ of straight-road driving for recovery. Fig. 16 shows the experimental scheme. Data for the first 10-min portion of each experiment were regarded as baseline data.
The subjects were expected to experience motion sickness during and after the 40-min winding-road driving session. The physiological signals collected during the "motion sickness" session were then compared with those in the "baseline" session. The VR scene was designed to simulate

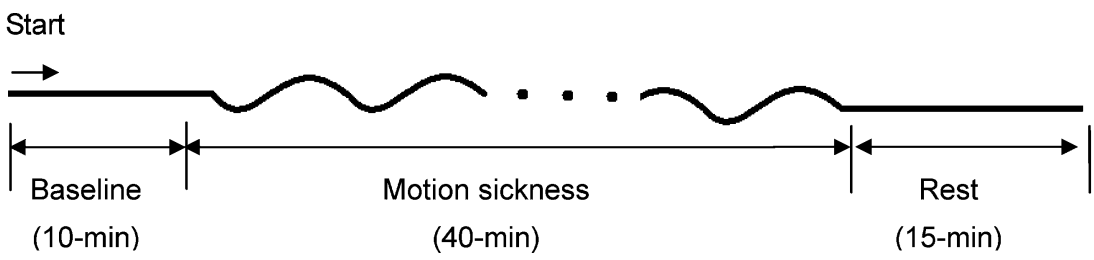

Fig. 16. Protocol of the designed VR scene to induce motion sickness. 


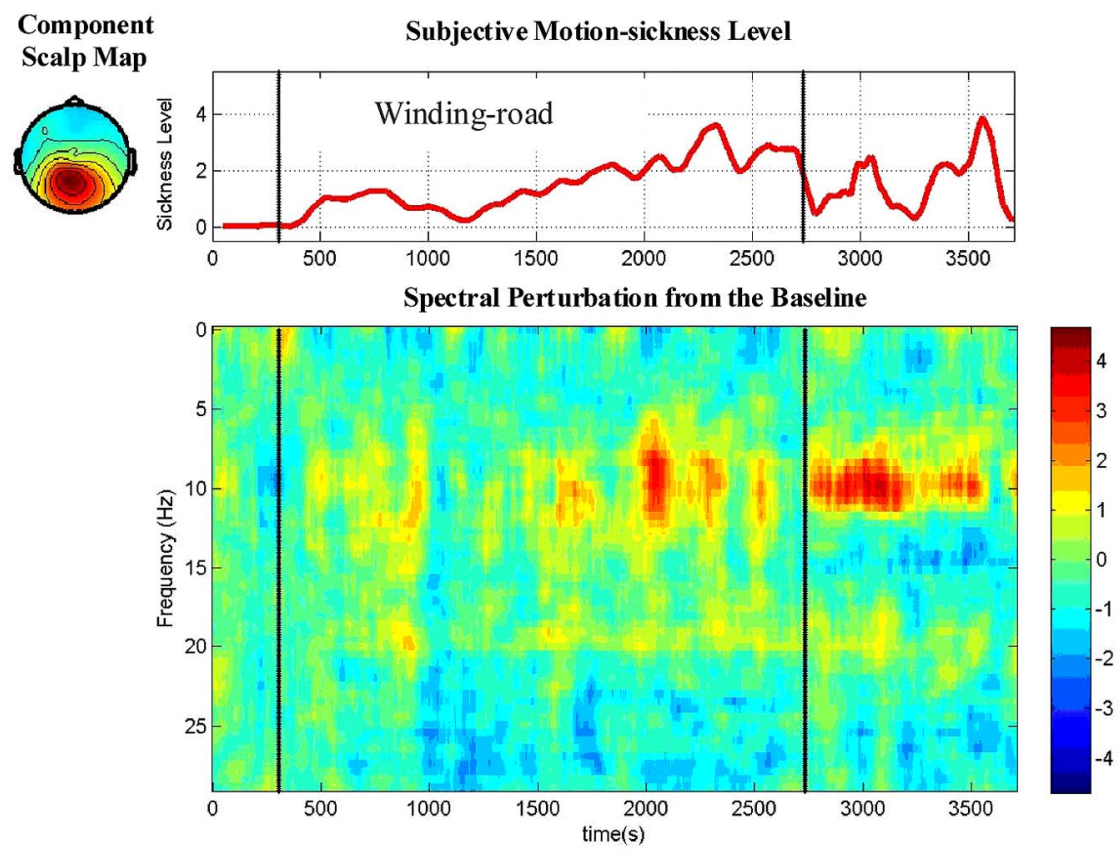

(a)

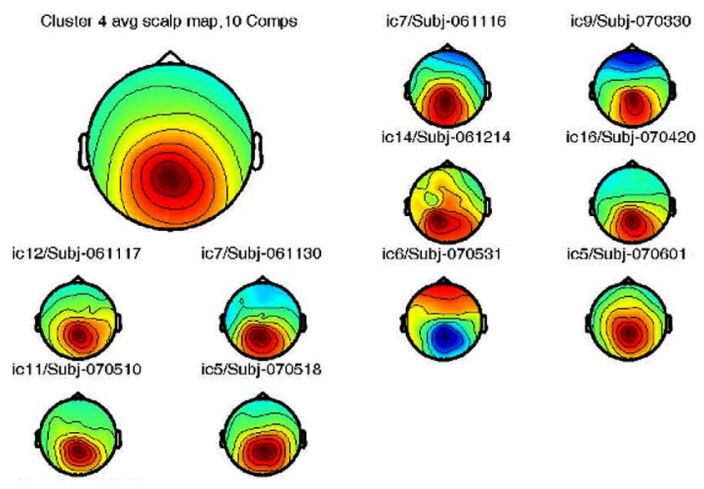

(b)

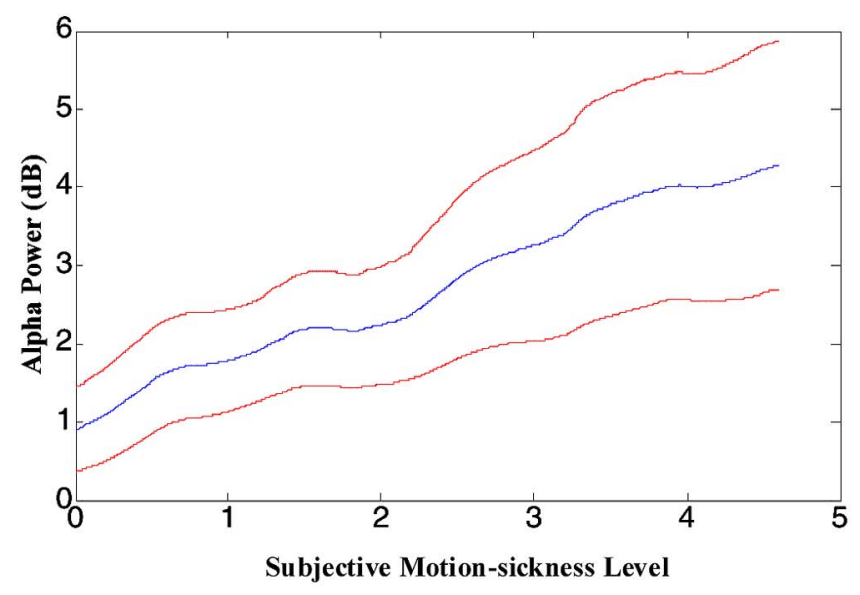

(c)

Fig. 17. The EEG correlates of motion sickness induced by a dynamic VR driving simulator. (a) ERSP of a sample independent component. (b) Mean and individual scalp maps of a centroparietal component cluster. (c) The mean log alpha power as a function of subjective motion-sickness level. 
driving in a tunnel, which is known to induce motion sickness.

Ten healthy volunteers (six males and four females; aged 18-26 years old; average age 22 years old) with no history of gastrointestinal, cardiovascular, or vestibular disorders participated in the experiments. All subjects had been instructed to avoid tobacco, caffeine, drugs, or alcohol for a week prior to the experiment to minimize influences on the central and autonomic nervous systems. A motion-sickness questionnaire was also designed based on several related studies to evaluate the behavioral performance of subjects during varying severity of motion sickness [44]. To fully explore the EEG correlates of motion sickness in the kinesthetic VR environment, this preliminary study employed a whole-head 32-channel EEG cap (Neuroscan, Inc.) to measure subject EEG data in the motion-sickness experiments. All channels were referenced to the right mastoid with input impedance lower than $5 \mathrm{k} \Omega$. Subjective sickness level was continuously reported by subjects using a joystick synchronized with EEG acquisition. After artifact removal, the EEG data were processed by independent component analysis [45][48] and time-frequency analysis to assess EEG correlates of motion sickness. Time series in each epoch $k$ were transformed into time-frequency matrix $F_{k}(f, t)$ using 1-s moving-window fast Fourier transforms. Log power spectra were estimated at 50 linear-spaced frequencies from 0.5 to $25 \mathrm{~Hz}$, then normalized by subtracting the log mean power spectrum in the baseline (first 10-min straight-road driving). The resulting event-related spectral perturbation (ERSP) images were constructed to show potentially significant spectral perturbations (log power differences) from the mean power spectral baseline ( $<<0.01$, not corrected for multiple comparisons) [49]. The power spectra of independent component (IC) time courses were also calculated and correlated with the subjective motion-sickness level to select motion-sicknessrelated IC(s).

\section{B. Results}

Fig. 17(a) shows the ERSP of a typical "nausea-related" independent component with an equivalent dipole source located in the centroparietal area. This component exhibited strong alpha-band $(8-12 \mathrm{~Hz})$ power increases when the subjects experienced motion sickness plotted above the ERSP image. The spectra of equivalent components of some subjects even exhibited parallel spectral increases at $18 \sim 20 \mathrm{~Hz}$. To test the consistency of component activities, clustering analysis of equivalent ICs was performed across sessions and subjects. The ICs from all experiments were grouped semiautomatically based on their scalp maps and power spectral baselines [50]. Fig. 17(b) shows the mean scalp map for the centroparietal component cluster. Individual maps in this cluster resembled the cluster mean map, indicating the component scalp projections, putatively equivalent dipole locations, and component activities were acceptably stable across sessions and subjects. Fig. 17(c) shows the mean (and +/- standard deviation) alpha power of the centroparietal components across sessions and subjects as a function of subjective motion-sickness level. Alpha band power increased monotonically with subjective motion-sickness level.

These experimental results suggest that continuous, accurate, noninvasive, and near real-time estimation of the physical state (in this example, motion sickness) of the driver is feasible using EEG measures recorded from the scalp.

\section{DISCUSSIONS AND CONCLUSIONS}

While most neural prosthetic systems to date have focused on invasive or implantable devices for patients with inoperative or malfunctioning body parts or organs, a much larger population of otherwise healthy people often suffer momentary, episodic, or progressive cognitive impairments in daily life and can benefit from noninvasive neural prosthesis. This paper proposes a mobile, wireless, and noninvasive prosthetic system that allows continuous monitoring of EEG and other physiological signals and prosthesis for monitoring physical and cognitive states. The unavailability of an EEG monitoring system capable of high-definition recording, online signal processing, and artifact cancellation without the need to apply conductive gels to the scalp has long limited the use of EEG monitoring in operational environments. The proposed mobile and wireless EEG system composed of dry MEMS EEG sensors, low-power signal acquisition, amplification and digitization, wireless telemetry, online artifact cancellation, and signal processing enables continuous and accurate monitoring of physiological signals in real operational environments. This paper also reports a dynamic VR motion platform integrating technologies of information science and electrical and mechanical control to gather multisensory (visual, auditory, kinesthetic, and tactile) information from participants in a safe yet realistic environment. The platform allows repetitive and systematic assessment of the complicated coupling between such information and the motor actions applied to the subjects and vehicle.

This paper also demonstrated the applications of this mobile and wireless noninvasive technology in neuroscience and neurotechnology through two case studies: 1) cognitive-state monitoring of participants performing realistic driving tasks in the VR-based dynamic driving simulator and 2) neural correlates of motion sickness in winding-road driving. The experimental results of these studies provide new insights into the understanding of complex brain functions of participants actively performing ordinary tasks in natural body positions and situations in operational environments. Such data would be difficult or impossible to obtain in a standard EEG 
laboratory where participants are asked to limit their eye blinks, teeth clenching, or other head/ body movements. These experimental results may also be applied in future studies to elucidate the limitations of normal human performance in repetitive task environments and may inspire more detailed study of changes in cognitive dynamics in brain-damaged, diseased, or genetically abnormal individuals. The proposed system has many potential applications in clinical research and practice in such diverse fields as neurology, psychiatry, gerontology, and rehabilitative medicine.

\section{Acknowledgment}

The authors would like to greatly thank Y. C. Chen, C. W. Chang, C. F. Chao, J. L. Jeng, Y. H. Lin, and S. W. Chuang for their developing and operating the experiments.

\section{REFERENCES}

[1] E. Margalit, M. Maia, J. Weiland, R. Greenberg, G. Fujii, G. Torres, D. Piyathaisere, T. O'Hearn, W. Liu, G. Lazzi, G. Dagnelie, D. Scribner, E. de Juan, Jr., and M. Humayun, "Retinal prosthesis for the blind," Surv. Ophthal., vol. 47, no. 4, pp. 335-356, 2002.

[2] B. S. Wilson, D. T. Lawson, J. M. Müller, R. S. Tyler, and J. Kiefer, "Cochlear implants: Some likely next steps,” Annu. Rev. Biomed. Eng., vol. 5, pp. 207-249, 2003.

[3] X. Navarro, T. B. Krueger, N. Lago, S. Micera, T. Stieglitz, and P. Dario, "A critical review of interfaces with the peripheral nervous system for the control of neuroprostheses and hybrid bionic systems," J. Periph. Nervous Syst., vol. 10, no. 3, pp. 229-258, 2005.

[4] S. Siegel, E. Paszkiewicz, C. Kirkpatrick, B. Hinkel, and K. Oleson, "Sacral nerve stimulation in patients with chronic intractable pelvic pain," J. Urol., vol. 166 no. 5, pp. 1742-1745, 2001.

[5] M. Mojarradi, D. Binkley, B. Blalock, R. Andersen, N. Ulshoefer, T. Johnson, and L. Del Castillo, "A miniaturized neuroprosthesis suitable for implantation into the brain," IEEE Trans. Neural Syst. Rehab. Eng., vol. 11, no. 1, pp. 38-42, 2003.

[6] N. H. Mackworth, "The breakdown of vigilance during prolonged visual search," Ergonomics, vol. 3, pp. 133-153, 2005.

[7] T. Pilutti and G. Ulsoy, "Identification of driver state for lane-keeping tasks," IEEE Trans. Syst., Man, Cybern. A, Syst. Humans, vol. 29, pp. 486-502, Sep. 1999.

[8] T. P. Jung, S. Makeig, M. Stensmo, and T. J. Sejnowski, "Estimating alertness from the EEG power spectrum," IEEE Trans. Biomed. Eng., vol. 44, no. 1, pp. 60-69, 1997.

[9] S. Makeig and T. P. Jung, "Changes in alertness are a principal component of variance in the EEG spectrum," Neuroreport, vol. 7, no. 1, pp. 213-216, 1995.

[10] H. J. Eoh, M. K. Chung, and S. H. Kim, "Electroencephalographic study of drowsiness in simulated driving with sleep deprivation," Int. J. Ind. Ergon., vol. 35, pp. 307-320, 2005.

[11] R. S. Huang, T. P. Jung, A. Delorme, and S. Makeig, "Tonic and phasic electroencephalographic dynamics during continuous compensatory tracking," Neuroimage, 2008, in press.

[12] N. G. Page and M. A. Gresty, "Motorist's vestibular disorientation syndrome," J. Neurol. Neurosurg. Psych., vol. 48, pp. 729-735, 1985.
[13] H. A. Miller and D. C. Harrison, Biomedical Electrode Technology. New York: Academic, 1974

[14] A. P. Spence, Basic Human Anatomy. Redwood City, CA: Benjamin Cumming, 1990.

[15] P. Griss, P. Enoksson, H. K. Tolvanen-Laakso, P. Merilainen, S. Ollmar, and G. Stemme, "Micromachined electrodes for biopotential measurements," J. Microelectromech. Syst., vol. 10, no. 1, pp. 10-16, 2001.

[16] A. P. Spence, Basic Human Anatomy. Redwood City, CA: Benjamin Cumming, 1990.

[17] J. G. Webster, Medical Instrumentation Application and Design, 3rd ed. New York: Wiley, 1998.

[18] J. C. Chiou, L. W. Ko, C. T. Lin, T. P. Jung, S. F. Liang, J. L. Jeng, and C. T. Hong, "Using novel MEMS EEG sensors in detecting drowsiness application," in Proc. IEEE Biomed. Circuits Syst. Conf. (BioCAS 2006), London, U.K., Nov. 29-Dec. 1, 2006.

[19] C. T. Lin, H. Y. Hsieh, L. W. Ko, M. Lin, S. F. Liang, and C. T. Hong, "Development of wireless brain computer interface with embedded multi-task scheduling," in Proc. IEEE Biomed. Circuits Syst. Conf. (BioCAS 2006), London, U.K., Nov. 29-Dec. 1, 2006.

[20] R. S. Huang, C. J. Kuo, L. L. Tsai, and O. T. C. Chen, "EEG pattern recognition arousal states detection and classification," in Proc. IEEE Int. Conf. Neural Netw., 1996, vol. 2, pp. 641-646.

[21] S. Makeig and M. Inlow, "Lapses in alertness: Coherence of fluctuations in performance and EEG spectrum," Electroencephal. Clin. Neurophysiol., vol. 86, pp. 23-35, 1993.

[22] A. Vuckovic, V. Radivojevic, A. C. N. Chen, and D. Popovic, "Automatic recognition of alertness and drowsiness from EEG by an artificial neural network," Med. Eng. Phys., vol. 24, pp. 349-360, 2002.

[23] S. Roberts, I. Rezek, R. Everson, H. Stone, S. Wilson, and C. Alford, "Automated assessment of vigilance using committees of radial basis function analysers," Proc. Inst. Elect. Eng. Sci., Meas. Technol., vol. 147, no. 6, pp. 333-338, Nov. 2000.

[24] K. B. Khalifa, M. H. Bedoui, R. Raytchev, and M. Dogui, "A portable device for alertness detection," in Proc. 1st Annu. Int. Conf. Microtechnol. Med. Biol., 2000, pp. 584-586.

[25] J. Wilson and T. D. Bracewell, "Alertness monitor using neural networks for EEG analysis," in Proc. IEEE Signal Process. Soc. Workshop Neural Netw. Signal Process., 2000 vol. 2, pp. 814-820.
[26] W. Wierville, J. G. Casali, and B. S. Repa, "Driver steering reaction time to abrupt-onset crosswind, as measured in a moving-base driving simulator," Human Factors, vol. 25, no. 1, pp. 103-116, 1983.

[27] G. Reymond, A. Kemeny, J. Droulez, and A. Berthoz, "Role of lateral acceleration in curve driving: Driver model and experiments on a real vehicle and a driving simulator," Human Factors, vol. 43, pp. 483-495, 2001.

[28] E. L. Groen, I. P. Howard, and B. S. Cheung, "Influence of body roll on visually induced sensation of self-tilt and rotation," Perception, vol. 28, pp. 287-297, 1999.

[29] C. T. Lin, I. F. Chung, L. W. Ko, Y. C. Chen, S. F. Liang, and J. R. Duann, "EEG-based assessment of driver cognitive responses in a dynamic virtual-reality driving environment," IEEE Trans. Biomed. Eng., vol. 54, no. 7, pp. 1349-1352, 2007.

[30] C. T. Lin, R. C. Wu, S. F. Liang, W. H. Chao, Y. J. Chen, and T. P. Jung, "EEG-based drowsiness estimation for safety driving using independent component analysis," IEEE Trans. Circuits Syst. I, Regular Papers, vol. 52, pp. 2726-2738, Dec. 2005.

[31] C. T. Lin, R. C. Wu, T. P. Jung, S. F. Liang, and T. Y. Huang, "Estimating alertness level based on EEG spectrum analysis," EURASIP J. Appl. Signal Process., vol. 2005, no. 19, pp. 3165-3174, Mar. 2005.

[32] R. S. Huang, T. P. Jung, and S. Makeig, "Multi-scale EEG brain dynamics during sustained attention tasks," in Proc. IEEE Int. Conf. Acoust., Speech Signal Process., 2007, vol. 4, pp. 1173-1176.

[33] N. V. Thakor, Biopotentials and ElectroPhysiology Measurement. Baltimore, MD: Johns Hopkins School of Medicine, 1999.

[34] M. Steriade, "Central core modulation of spontaneous oscillations and sensory transmission in thalamocortical systems," Current Opinion Neurobiol., vol. 3, no. 4, pp. 619-625, 1993.

[35] S. Chatterjee and A. S. Hadi, "Influential observations, high leverage points, and outliers in linear regression," Statist. Sci., pp. 379-416, 1986.

[36] C. M. Bishop, Neural Networks for Pattern Recognition. Oxford, U.K.: Oxford Univ. Press, 1995.

[37] S. Hu, K. A. McChesney, K. A. Player, A. M. Bahl, J. B. Buchanan, and J. E. Scozzafava, "Systematic investigation of physiological correlates of motion sickness induced by viewing an optokinetic rotating drum," Aviation, Space, Environ. Med., vol. 70, no. 8, pp. 759-765, 1999. 
[38] B. Cheung and P. Vaitkus, "Perspectives of electrogastrography and motion sickness," Brain Res. Bull., vol. 47, no. 5 , pp. 421-431, 1998.

[39] J. T. Reason and J. J. Brand, Motion Sickness. London, U.K.: Academic, 1975.

[40] G. E. Riccio and T. A. Stoffregen, "An ecological theory of motion sickness and postural instability," Ecol. Psychol., vol. 3, no. 3, pp. 195-240, 1991.

[41] T. Russomano, D. F. G. de Azevedo, L. Piedade, F. S. Glock, M. Tello, M. A. dos Santos, F. P. Falcao, and J. L. Giongo, "Development and validation of an electrically controlled rotatory chair to be used as a simulator for spatial disorientation and motion sickness," in Proc. 25th Annu. Int. Conf. IEEE Eng. Med. Biol. Soc., 2003, vol. 4, pp. 3306-3308.

[42] M. J. Williamson, M. J. Thomas, and R. M. Stern, "The contribution of expectations to motion sickness symptoms and gastric activity," J. Psychosom. Res., vol. 56, no. 6, pp. 721-726, 2004.

[43] S. J. Wood, "Human otolith-ocular reflexes during off-vertical axis rotation: Effect of frequency on tilt-translation ambiguity and motion sickness," Neurosci. Lett., vol. 323, no. 1, pp. 41-44, 2002.

[44] R. S. Kennedy, N. E. Lane, K. S. Berbaum, and M. G. Lilienthal, "Simulator sickness questionnaire: An enhanced method for quantifying simulator sickness," Int. J. Aviation Psychol., vol. 3, pp. 203-220, 1993.

[45] A. J. Bell and T. J. Sejnowski, "An information-maximization approach to blind separation and blind deconvolution," Neural Comput., vol. 7, pp. 1129-1159, 1995.

[46] S. Makeig, A. J. Bell, T. P. Jung, and T. J. Sejnowski, "Independent component analysis of electroencephalographic data," in Advances in Neural Information Processing Systems, D. Touretzky, M. Mozer, and M. Hasselmo, Eds. Cambridge, MA: MIT Press, 1996, vol. 8, pp. 145-151.
[47] S. Makeig, T.-P. Jung, D. Ghahremani, A. J. Bell, and T. J. Sejnowski, "Blind separation of auditory event-related brain responses into independent components," Proc. Nat. Acad. Sci. USA, vol. 94, pp. 10979-10984, 1997.

[48] T. P. Jung, S. Makeig, M. J. Mckeown, A. J. Bell, T. W. Lee, and T. J. Sejnowski, "Imaging brain dynamics using independent component analysis," Proc. IEEE, vol. 89, no. 7, pp. 1107-1122, 2001.

[49] S. Makeig, "Auditory event-related dynamics of the EEG spectrum and effects of exposure to tones," Electroenceph. Clin. Neurophys., vol. 86, pp. 283-293, 1993.

[50] T. P. Jung, S. Makeig, M. Westerfield, J. Townsend, E. Courchesne, and T. J. Sejnowski, "Analysis and visualization of single-trial event-related potentials," Human Brain Map., vol. 14, pp. 166-185, 2001.

\section{ABOUT THE AUTHORS}

Chin-Teng (CT) Lin (Fellow, IEEE) received the B.S. degree from National Chiao-Tung University (NCTU), Taiwan, R.O.C., in 1986 and the Ph.D. degree in electrical engineering from Purdue University, West Lafayette, IN, in 1992.

$\mathrm{He}$ is currently the Chair Professor of Electrical and Computer Engineering, Dean of Academic Affairs, and Director of the Brain Research Center at NCTU. He is coauthor of Neural Fuzzy Systems-A Neuro-Fuzzy Synergism to Intelligent Systems (Englewood Cliffs, NJ: Prentice Hall, 1996) and the author of Neural Fuzzy Control Systems with Structure and Parameter Learning (Singapore: World Scientific, 1994). He is an Associate Editor of the International Journal of Speech Technology.

Dr. Lin was a Distinguished Lecturer of the IEEE Circuits and Systems Society from 2003 to 2005. He has been President of the Asia Pacific Neural Network Assembly since 2004. He was named one of the 38th Ten Outstanding Rising Stars in Taiwan (2000). He currently is an Associate Editor of IEeE TRansactions on Circuits and Systems-Part I: Regular Papers and Part II: Analog and Digital Signal Processing; IEeE Transactions on Systems, man, and Cybernetics, ieeE transactions on Fuzzy SYSTEMS.

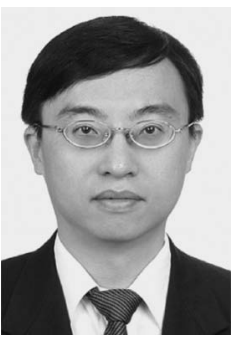
Jin-Chern Chiou (Member, IEEE) received the
M.S. and Ph.D. degrees in aerospace engineering science from the University of Colorado at Boulder, Boulder, in 1986 and 1990, respectively.

Before joining the Department of Electrical and Control Engineering, National Chiao-Tung University (NCTU), Taiwan, R.O.C., in 1992, he was with the Center for Space Structure and Control, University of Colorado, as a Research Associate (1991-1992). His research interests include micro-

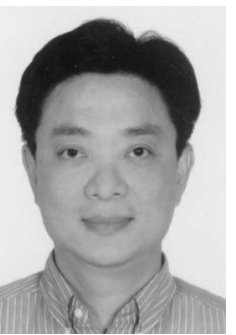
electromechanical systems (MEMS), biomedical chips, gas sensors, and modeling and control of multibody dynamic systems. He has published more than 50 journal papers. He is the coauthor of advanced reference books on MEMS technology and application, CD-ROM system technology, and mechanics and control of large flexible structures. He has received five U.S. patents with three pending and eight R.O.C. patents with three pending.

Dr. Chiou has received several awards from the Acer Foundation, Y. Z. Hsu Foundation, Taiwan Information Storage Association, Institute for Biotechnology and Medicine Industry, NCTU, and National Science Council, R.O.C., for his outstanding MEMS, biomedical device, and control research.
Li-Wei Ko received the B.S. degree in mathematics from National Chung Cheng University, Chiayi, Taiwan, R.O.C., in 2001, the M.S. degree in educational measurement and statistics from National Taichung University, Taichung, Taiwan, in 2004, and the Ph.D. degree in electrical and control engineering from National Chiao-Tung University (NCTU), Hsinchu, Taiwan, in 2007.

$\mathrm{He}$ is currently an Executive Officer with the Brain Research Center, NCTU. His research interests are in the areas of machine learning, brain computer interface, cognitive neural engineering, and biomedical signal processing.

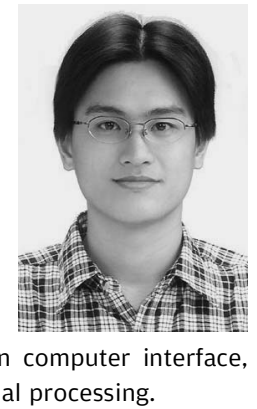

Jeng-Ren Duann (Member, IEEE) received the B.S. and M.S. degrees in biomedical engineering and the Ph.D. degree in physics from Chung Yuan University, Taiwan, R.O.C., in 1990, 1992, and 1999, respectively.

He was a Research Associate with the Computational Neurobiology Laboratory, The Salk Institute, San Diego, CA. He is currently an Assistant Project Scientist with the Institute for Neural Computation, University of California, San Diego.

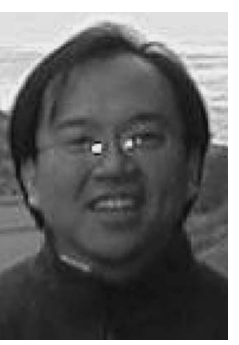
$\mathrm{He}$ is the author of FMRLAB, a freely downloadable Matlab toolbox for functinal neuroimaging data analysis using independent component analysis. His research interests are in the areas of biomedical signal and image processing, biosystem simulation and modeling, structural and functional human brain mapping and applications in cognitive neuroscience, and functional cardiac imaging. 
Ruey-Song Huang received the B.S. and M.S. degrees in electrical engineering from National Chung Cheng University, Chiayi, Taiwan, R.O.C., in 1996 and 1998, respectively, and the M.S. and Ph.D. degrees in cognitive science from the University of California San Diego (UCSD), La Jolla, CA, in 2003 and 2006, respectively.

He is currently a Postdoctoral Fellow with the Swartz Center for Computational Neuroscience, UCSD. His research interests are in the areas of

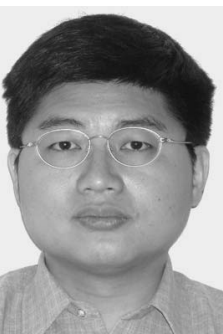
cognitive neuroscience, human brain mapping, and multimodal neuroimaging ( $\mathrm{MRI}$ and EEG).

Sheng-Fu Liang was born in Tainan, Taiwan, R.O.C., in 1971. He received the B.S. and M.S. degrees in control engineering and the Ph.D. degree in electrical and control engineering from National Chiao-Tung University (NCTU), Taiwan, in 1994, 1996, and 2000, respectively.

From 2001 to 2005, he was a Research Assistant Professor in the Department of Electrical and Control Engineering, NCTU. In 2005, he joined the Department of Biological Science and Tech-

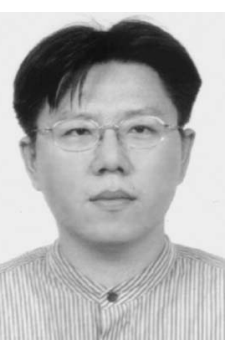
nology, NCTU, where he was an Assistant Professor. Currently, he is an Assistant Professor in the Department of Computer Science and Information Engineering, National Cheng-Kung University, Tainan, and a Collaborative Researcher with the Brain Research Center, NCTU. His current research interests are biomedical engineering, biomedical signal/image processing, machine learning, and multimedia signal processing.
Tzai-Wen Chiu received the B.S. degree in nursing from National Taiwan University, Taipei, Taiwan, R.O.C., in 1995 and the M.S. and Ph.D. degrees in physiology from the Medical College, National Cheng Kung University, Tainan, Taiwan, in 1997 and 2004, respectively.

From 2005 to 2006, she was a Research Associate with the Bat Laboratory, Department of Psychology, Art and Science College, University of Washington, Seattle. In 2006, she joined the

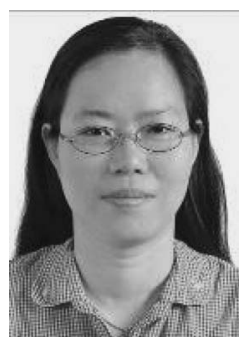
Brain Research Center, National Chiao-Tung University, as a Research Assistant Professor. Her research interests are biosignal processing, cognitive neuroscience, neural plasticity, neural mechanisms of sound coding, and tinnitus.

Tzyy-Ping Jung (Senior Member, IEEE) received the B.S. degree in electronics engineering from National Chiao-Tung University, Taiwan, R.O.C., in 1984 and the M.S. and Ph.D. degrees in electrical engineering from The Ohio State University, Columbus, in 1989 and 1993, respectively.

He was a Research Associate with the Computational Neurobiology Laboratory, The Salk Institute, San Diego, CA. He is currently an Associate Research Scientist with the Institute for Neural Computation, University of California San Diego. He is also Associate Director of the Swartz Center for Computational Neuroscience, UCSD. His research interests are in the areas of biomedical signal processing, cognitive neuroscience, machine learning, time-frequency analysis of human EEG, functional neuroimaging, and brain-computer interfaces and interactions.

Dr. Jung received the 2008 ICA Unsupervised Learning Pioneer Award from the Society for Photo-Optical Instrumentation Engineers in 2008. 